\title{
Incidence, Severity, and Outcomes of Acute Kidney Injury in Octogenarians following Heart Valve Replacement Surgery
}

\author{
Michael A. Mao, ${ }^{1}$ Charat Thongprayoon, ${ }^{2}$ YiFan Wu, ${ }^{1}$ Vickram Tejwani, ${ }^{1}$ \\ Myriam Vela-Ortiz, ${ }^{1}$ Joseph Dearani, ${ }^{3}$ and Qi Qian ${ }^{1}$ \\ ${ }^{1}$ Division of Nephrology and Hypertension, Department of Medicine, Mayo Clinic College of Medicine, Rochester, MN 55905, USA \\ ${ }^{2}$ Division of Anesthesiology, Mayo Clinic College of Medicine, Rochester, MN 55905, USA \\ ${ }^{3}$ Department of Surgery, Mayo Clinic College of Medicine, Rochester, MN 55905, USA \\ Correspondence should be addressed to Qi Qian; qian.qi@mayo.edu
}

Received 12 January 2015; Revised 5 April 2015; Accepted 23 April 2015

Academic Editor: Danuta Zwolinska

Copyright (C) 2015 Michael A. Mao et al. This is an open access article distributed under the Creative Commons Attribution License, which permits unrestricted use, distribution, and reproduction in any medium, provided the original work is properly cited.

\begin{abstract}
Background. The study investigates the occurrence, severity, and outcomes of acute kidney injury (AKI) in octogenarians following heart valve surgery. Methods. All patients, age $>80$ years, not on dialysis and without kidney transplant, undergoing heart valve replacement at Mayo Clinic, Rochester, in the years 2002-2003 were enrolled. AKI was diagnosed based on AKIN criteria. Results. 209 octogenarians ( $88.0 \%$ aortic valve, $6.2 \%$ mitral valve, $1.0 \%$ tricuspid valve, and $4.8 \%$ multivalve) with (58.4\%) and without CABG were studied. 34 (16.3\%) had preexisting CKD. After surgery, 98 (46.8\%) developed AKI. 76.5\% of the AKI were in Stage 1, 9.2\% in Stage 2, and $14.3 \%$ in Stage 3. 76.5\% CKD patients developed AKI. Length of hospital stay was longer for AKI patients. More AKI patients were discharged to care facilities. Patient survival at 30 days and 1 year for AKI versus non-AKI was 88.8 versus $98.7 \%$, $p=0.003$, and 76.5 versus $88.3 \%, p=0.025$, respectively. With follow-up of $3.94 \pm 0.28$ years, Kaplan-Meier analysis showed a reduced survival for AKI octogenarians. Preexisting CKD and large volume intraoperative fluid administration were independent AKI predictors. Conclusions. Nearly half of the octogenarians developed AKI after valve replacement surgery. AKI was associated with significant functional impairment and reduced survival.
\end{abstract}

\section{Introduction}

Population is aging in the United States and nearly worldwide [1]. The US Census Bureau projects that the very elderly population (age $\geq 80$ ) will grow from 5.8 million (1.8\% of the population) in 2012 to 13 million (3.2\%) by 2050 [2]. Heart valves are well known to degenerate with aging, affecting up to $13.2 \%$ patients age $\geq 75$ years [3]. With limited nonsurgical treatment options for valvular heart diseases and significant clinical morbidity and mortality, it is foreseeable that valve replacement operations will be performed for more elderly in the future [3-7]. With limited organ function reserve, increasing comorbidities, and reduced adaptive capacity, the elderly are at high risk for postoperative complications such as AKI [8]. Aging alone is a significant risk factor for AKI [9], and the severity of AKI is proportional to the poor outcomes including morbidity and mortality [10]. Data on the incidence, severity, and outcomes of AKI in octogenarians undergoing heart valve surgery is, however, scarce [5].

The aim of this study is to determine AKI occurrence, severity, and outcomes in a cohort of octogenarians undergoing valve replacement surgery. We also explore potential predictors for the development of AKI in this cohort.

\section{Patients and Methods}

2.1. Data Collection and AKI Definitions. The Institutional Review Board approved the study. Between 2002 and 2003, 210 octogenarians with symptomatic cardiac valve disease underwent valve replacement surgery who were not on hemodialysis and without kidney transplant. The Charlson Comorbidity Index at the time of admission was collected for each patient [11]. Survival was censored based on death dates in the institutional records and publically 
accessible Social Security Death Index http://www.genealogybank.com/gbnk/ssdi/.

Preexisting chronic kidney disease (CKD) was defined based on primary physician's documentation within six months of the surgery. Baseline serum creatinine (s.Cr, in $\mathrm{mg} / \mathrm{dL}$ ) was defined as the s.Cr measurement within three months of the index admission. Estimated GFR was calculated based on the Chronic Kidney Disease Epidemiology Collaboration (CKD-EPI) [12]. The patients were grouped based on their eGFR. One patient who expired intraoperatively was excluded from analysis.

Acute kidney injury (AKI) was defined as an abrupt (within 48 hours) increase in s.Cr $\geq 0.3 \mathrm{mg} / \mathrm{dL}$ or s.Cr increase by $50 \%$ from baseline within seven days postoperatively. The AKIN urinary criteria were omitted due to incomplete data on urine output [13]. The severity of AKI was determined using s.Cr definitions from the AKIN criteria [13]. The AKIN criteria classify AKI into the following: Stage 1, s.Cr elevation $\geq 1.5$ times the baseline s.Cr; Stage $2, \geq 2$ times the baseline s.Cr; Stage 3, $\geq 3$ times the baseline s.Cr, s. Cr $\geq 4.0 \mathrm{mg} / \mathrm{dL}$ or complete loss of kidney function requiring renal replacement therapy.

Net fluid balance equaled to fluid input minus fluid output (in liters).

2.2. Statistical Analysis. Statistical analysis was performed using JMP version 10.0.0. For categorical variables, Fisher's exact test and Pearson's chi-squared test were utilized. For continuous variables, Student's $t$-test was applied for comparison between the AKI and non-AKI cohorts. The results are reported as percentage frequencies and means \pm standard deviation (SD). $p$ value of $<0.05$ (two-tailed) was considered significant. Stepwise backward logistic regressions were performed to derive the final multivariate model taking into consideration colinearity, interaction, and number of patients who expressed the outcome of interest. Cox proportional hazard regression modeling was used to compare survival in the two cohorts adjusted for age and comorbidity. Kaplan-Meier analysis was used to compare the long-term survival.

\section{Results}

3.1. Patient Characteristics. The 209 octogenarians represented $16 \%$ of the total patients undergoing valve replacement surgery at Mayo Clinic Rochester in 2002 and 2003. 184 $(88.0 \%)$ of the 209 underwent aortic valve replacement (AVR), 13 (6.2\%) mitral valve replacement (MVR), 2 (1.0\%) tricuspid valve replacement (TVR), and $10(4.8 \%)$ combined valve replacement (6 AV-MV, $1 \mathrm{AV}-\mathrm{TV}, 2 \mathrm{MV}-\mathrm{TV}$, and 1 PV-TV). All but one patient had bioprosthetic replacement valves. Preoperative characteristics of the cohort showed a median age of 84.1 years and a mean Charlson comorbidity score [11] of $2.63 \pm 1.76$ (Table 1). 127 (60.8\%) patients had preexisting hypertension and $35(16.7 \%)$ patients had a prior myocardial infarction. Thirty-four (16.3\%) patients had a diagnosis of CKD with a mean s.Cr of $1.7 \pm 0.31 \mathrm{mg} / \mathrm{dL}$ corresponding to an eGFR of $34.3 \pm 10.0 \mathrm{~mL} / \mathrm{min} / \mathrm{BSA}$,
TABle 1: Preoperative characteristics of octogenarians.

\begin{tabular}{lc}
\hline Baseline characteristics & $N=209$ \\
\hline Age (year) at surgery, median [range] & $84.1[80.1-93.4]$ \\
Males (\%) & $115(55.0)$ \\
& Caucasian $175(83.7)$ \\
Race (\%) & Other: $32(15.3)$ \\
& Black: $1(0.48)$ \\
BMI, mean \pm SD & $27.7 \pm 5.3$ \\
HTN (\%) & $127(60.8)$ \\
Diabetes (\%) & $40(19.1)$ \\
CKD (\%) & $34(16.3)$ \\
CHF (\%) & $167(79.9)$ \\
Prior MI (\%) & $35(16.7)$ \\
Hyperlipidemia on lipid-lowering agents (\%) & $104(49.8)$ \\
Charlson Index, mean \pm SD & $2.63 \pm 1.76$ \\
Baseline Cr, mean \pm SD & $1.24 \pm 0.30$ \\
eGFR, mean \pm SD & $49.79 \pm 12.79$ \\
Pre-op EF \%, mean \pm SD & $54.6 \pm 14.9$ \\
\hline
\end{tabular}

whereas 175 non-CKD patients had s.Cr of $1.15 \pm 0.20 \mathrm{mg} / \mathrm{dL}$, eGFR of $52.8 \pm 11.0 \mathrm{~mL} / \mathrm{min} / \mathrm{BSA}, p<0.0001$ (Table 2).

3.2. AKI Occurrence and Severity. Ninety-eight of 209 patients (46.8\%) developed postoperative AKI, of which 13 (6.2\%) required renal replacement therapy (7 from the CKD group and 6 from the non-CKD group). The majority of AKIs were within AKIN Stage 1 (risk). Patients with preexisting CKD were at higher risk for postoperative AKI, 26 (76.5\%) versus $72(41.1 \%)$ in non-CKD, $p=0.0002$. AKI in CKD patients were more severe, $26.9 \%$ versus $9.7 \%$ in Stage 3 (Table 2).

Prior studies show that, among octogenarians undergoing AVR, age $\geq 84$ years had the highest odds ratio for 6month mortality [14]. We examined AKI occurrence in octogenarians age $<84$ and $\geq 84$. The eGFR was different, $52.5 \mathrm{~mL} / \mathrm{min} / \mathrm{BSA}$ in those age $<84(N=101)$ and $47.2 \mathrm{~mL} / \mathrm{min} / \mathrm{BSA}$ in age $\geq 84(N=108), p=0.003$. However, the AKI occurrence between the two age groups did not differ, 44.4 versus $49.5 \%, p>0.05$. The proportion of AKI patients in Stages 1, 2, and 3 was also not different in the two age groups. The 6-month mortality rate was likewise comparable, 12.9 versus $12.0 \%, p>0.05$.

We also compared those with and without concomitant coronary artery bypass grafting (CABG), as previous studies had shown conflicting results on the effect of concomitant CABG on mortality $[5,7,14]$. In our cohort, CABG did not increase postoperative AKI. One-year mortality rate in the two groups was comparable, $13.9 \%$ with CABG versus $21.8 \%$ without CABG, $p=0.14$. The number of bypassed vessels did not affect the 30-day, 6-month, or 1-year mortality (data not shown).

3.3. Characteristics of AKI versus Non-AKI Patients. Patient characteristics in AKI and non-AKI were compared. AKI patients tended to have preexisting CKD, prior myocardial 
TABLE 2: AKI occurrence and severity in CKD and non-CKD patients.

\begin{tabular}{|c|c|c|c|c|}
\hline Baseline CKD status & $\begin{array}{c}\text { All } \\
n=209\end{array}$ & $\begin{array}{l}\text { CKD } \\
n=34\end{array}$ & $\begin{array}{c}\text { Non-CKD } \\
n=175\end{array}$ & $p$ value \\
\hline Presurgery s.Cr, mg/dL & $1.24(0.30)$ & $1.70 \pm 0.31$ & $1.15 \pm 0.20$ & $<0.0001$ \\
\hline Presurgery eGFR & $49.8(12.8)$ & $34.3 \pm 10.0$ & $52.8 \pm 11.0$ & $<0.0001$ \\
\hline \multicolumn{5}{|l|}{ Calculated CKD stage* } \\
\hline CKD Stage 1 & 0 & 0 & 0 & \\
\hline CKD Stage 2 & $45(21.5)$ & $1(2.9)$ & $44(25.1)$ & 0.004 \\
\hline CKD Stage 3a & $93(44.5)$ & $4(11.8)$ & $89(50.9)$ & $<0.0001$ \\
\hline CKD Stage $3 b$ & $57(27.3)$ & $17(50.0)$ & $40(22.9)$ & 0.0011 \\
\hline CKD Stage 4 & $14(6.7)$ & $12(35.3)$ & $2(1.1)$ & $<0.0001$ \\
\hline CKD Stage 5 & 0 & 0 & 0 & \\
\hline Total AKI number (\%) & $98(46.8)$ & $26(76.5)$ & $72(41.1)$ & 0.0002 \\
\hline Stage 1 & $75(76.5)$ & $17(65.4)$ & $58(80.6)$ & \\
\hline Stage 2 & $9(9.2)$ & $2(7.7)$ & $7(9.7)$ & 0.0001 \\
\hline Stage 3 & $14(14.3)$ & $7(26.9)^{\mathrm{a}}$ & $7(9.7)^{\mathrm{b}}$ & \\
\hline
\end{tabular}

${ }^{\mathrm{a}} 7$ of 7 in CKD and ${ }^{\mathrm{b}} 6$ of 7 in non-CKD required dialysis.

${ }^{*}$ Stratified by eGFR instead of physician's diagnosis of CKD.

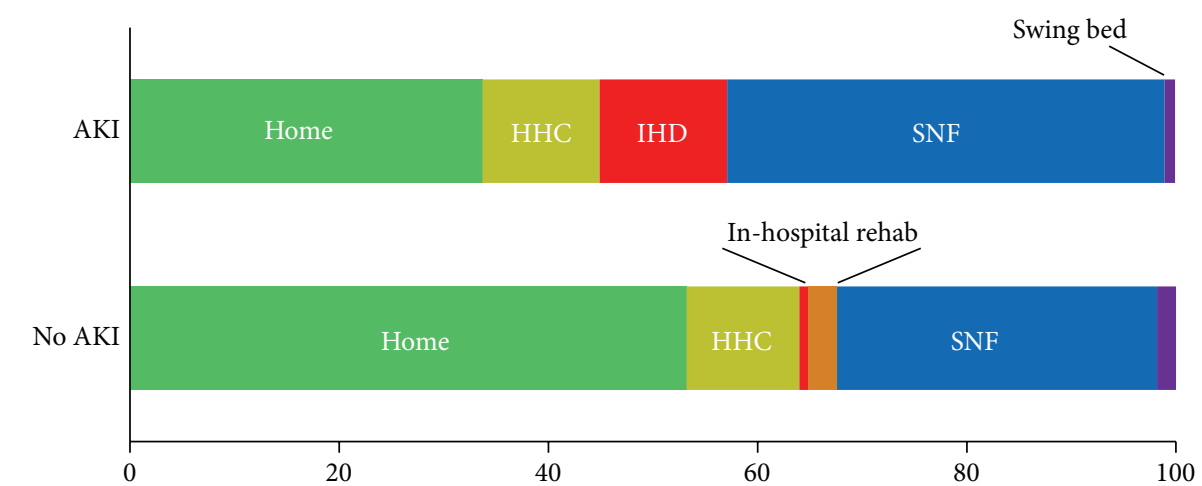

(\%)

\begin{tabular}{cccccccc}
\hline Count & Home & $\begin{array}{c}\text { Home health } \\
\text { care (HHC) }\end{array}$ & $\begin{array}{c}\text { In-hospital } \\
\text { death (IHD) }\end{array}$ & $\begin{array}{c}\text { In-hospital } \\
\text { rehab (IHR) }\end{array}$ & $\begin{array}{c}\text { Skilled } \\
\text { nursing } \\
\text { facility (SNF) }\end{array}$ & Swing bed & Total \\
\hline AKI $(n=98)$ & $33(33.7 \%)$ & $11(11.2 \%)$ & $12(12.2 \%)$ & 0 & $41(41.8 \%)$ & $1(1.0 \%)$ & 98 \\
No AKI $(n=111)$ & $59(53.2 \%)$ & $12(10.8 \%)$ & $1(0.9 \%)$ & $3(2.7 \%)$ & $34(30.6 \%)$ & $2(1.8 \%)$ & 111 \\
\hline
\end{tabular}

Figure 1: Patient disposition.

infarction, higher Charlson Comorbidity Index, and lower preoperative hemoglobin (Table 3 ). Intraoperative factors associated with AKI included longer surgical duration, intra-aortic balloon pump use, lower intraoperative nadir hemoglobin, and higher volume of blood transfusion and fluids/saline infusion (Table 3). Additionally, AKI patients were possibly more likely to have a higher BMI and atherosclerotic vascular diseases, although $p$ value did not reach statistical significance.

3.4. Predictors for AKI. Potential AKI predictors in this cohort of octogenarians were explored. Univariate analysis showed prior MI, CKD, higher Charlson Comorbidity Index, lower preoperative hemoglobin, longer surgical duration, blood transfusion, and intraoperative fluid and saline administration as potential risk factors. Two models of multivariate stepwise backward logistic regression analysis were built. Preexisting CKD (OR 4.87-5.38, 95\% CI 2.12-13.6, $p<$ 0.001 ), intraoperative fluid administration (OR 1.10, CI 1.01$1.21, p=0.03$ ) and intraoperative saline infusion (OR 1.52, CI 1.05-2.24, $p=0.02$ ) were shown as independent predictors for AKI (Tables 4(a) and 4(b)).

3.5. Length of Hospital Stay and Disposition. Length of hospital stay (LOS) was longer for AKI patients $14.0 \pm 11.7$ days versus $9.2 \pm 4.2$ days for non-AKI patients, $p<0.0001$. Patient disposition was different in octogenarians with and without AKI (Figure 1, $p=0.001$ ). More AKI patients discharged 
TABLE 3: Preoperative characteristics and hospital course in AKI and non-AKI octogenarians.

\begin{tabular}{|c|c|c|c|}
\hline Preoperative characteristics & $\begin{array}{c}\text { No AKI, mean } \pm \mathrm{SD} \text {, } \\
n=111\end{array}$ & $\begin{array}{c}\text { AKI, mean } \pm \mathrm{SD}, \\
n=98\end{array}$ & $p$ value \\
\hline Age, median [range] & $84.1 \pm 2.8[80.1-93.4]$ & $\begin{array}{c}83.9 \pm 2.8 \\
{[80.1-92.0]}\end{array}$ & 0.49 \\
\hline Sex, male (\%) & $57(51.3)$ & $58(59.2)$ & 0.26 \\
\hline BMI, $\mathrm{kg} / \mathrm{m}^{2}$ & $27.1 \pm 4.9$ & $28.4 \pm 5.7$ & 0.09 \\
\hline HTN (\%) & $72(64.9)$ & $55(56.1)$ & 0.20 \\
\hline Diabetes $(\%)$ & $18(16.2)$ & $22(22.4)$ & 0.25 \\
\hline Hyperlipidemia on lipid-lowering agents (\%) & $62(55.9)$ & $42(42.9)$ & 0.06 \\
\hline Chronic pulmonary disease (\%) & $19(17.1)$ & $14(14.3)$ & 0.58 \\
\hline Preexisting CKD (\%) & $8(7.2)$ & $26(26.5)$ & $<0.0001$ \\
\hline Prior myocardial infarction (\%) & $12(10.8)$ & $23(23.5)$ & 0.01 \\
\hline $\mathrm{CAD}(\%)$ & $78(70.3)$ & $65(66.3)$ & 0.54 \\
\hline Peripheral vascular, cerebrovascular, or carotid artery disease (\%) & $29(26.1)$ & $36(36.7)$ & 0.10 \\
\hline $\mathrm{CHF}(\%)$ & $94(84.7)$ & $73(74.5)$ & 0.07 \\
\hline Charlson Comorbidity Index & 2.39 & 2.91 & 0.03 \\
\hline Pre-op EF, \% & $53.7 \pm 15.5$ & $55.7 \pm 14.3$ & 0.34 \\
\hline Pre-op hemoglobin, g/dL & $12.8 \pm 1.6$ & $11.9 \pm 1.6$ & 0.03 \\
\hline \multicolumn{4}{|l|}{ Hospital course } \\
\hline Surgical duration, min & $331.0 \pm 82.8$ & $369.1 \pm 108.5$ & 0.005 \\
\hline CABG, yes or no $(\%)$ & $64(57.7)$ & $58(59.2)$ & 0.82 \\
\hline $\operatorname{IABP}(\%)$ & $2(1.80)$ & $9(9.18)$ & 0.02 \\
\hline Cross clamp time, min & $69.7 \pm 30.1$ & $69.0 \pm 32.3$ & 0.85 \\
\hline $\mathrm{CPB}, \min$ & $96.7 \pm 39.3$ & $101.3 \pm 43.3$ & 0.42 \\
\hline Net intraoperative fluid balance, $\mathrm{L}$ & $6.87 \pm 2.57$ & $8.12 \pm 4.28$ & 0.01 \\
\hline Total intraoperative saline, $\mathrm{L}$ & $0.90 \pm 0.72$ & $1.16 \pm 0.95$ & 0.02 \\
\hline Intraoperative hemoglobin, $\mathrm{g} / \mathrm{dL}^{\mathrm{a}}$ & $8.28 \pm 1.04$ & $7.92 \pm 1.04$ & 0.01 \\
\hline $\mathrm{RBC}$ on operative day, $\mathrm{L}$ & $0.74 \pm 0.63$ & $1.06 \pm 1.03$ & 0.008 \\
\hline $\mathrm{RBC}$ during hospitalization, $\mathrm{L}$ & $0.92 \pm 0.86$ & $1.54 \pm 1.65$ & 0.0007 \\
\hline Length of hospital stay, day & $9.2 \pm 4.2(n=110)$ & $14.0 \pm 11.7(n=86)$ & $<0.0001$ \\
\hline Discharge creatinine, mg/dL & $1.09 \pm 0.25$ & $1.46 \pm 0.56$ & $<0.0001$ \\
\hline
\end{tabular}

${ }^{a}$ The lowest hemoglobin during the operation.

TABLE 4: Multivariate stepwise logistic regression models for predictors of postoperative AKI.

(a) Model number 1

\begin{tabular}{lcc}
\hline Variables & OR $(95 \% \mathrm{CI})$ & $p$ value \\
\hline CHF & $0.43(0.21-0.88)$ & 0.02 \\
CKD & $4.87(2.12-12.3)$ & $<0.001$ \\
Intraoperative fluid volume & $1.10(1.01-1.21)$ & 0.03 \\
\hline
\end{tabular}

(b) Model number 2

\begin{tabular}{lcc}
\hline Variables & OR $(95 \%$ CI $)$ & $p$ value \\
\hline CHF & $0.43(0.21-0.87)$ & 0.02 \\
CKD & $5.38(2.34-13.6)$ & $<0.001$ \\
Intraoperative saline volume & $1.52(1.05-2.24)$ & 0.02 \\
\hline
\end{tabular}

to skilled nursing facilities (41.8\% AKI versus $30.6 \%$ non$\mathrm{AKI}$ ) and fewer to home (33.7\% AKI versus $53.2 \%$ non-AKI), reflecting a higher level of functional impairment in patients with AKI, which is known to be associated with a high longterm mortality rate [15]. The disposition to a swing bed, another hospital adjacent to patient's home, was not different between the AKI and non-AKI groups.

3.6. Renal and Patient Outcomes. Among surviving AKI patients, s.Cr at the time of hospital discharge was higher in AKI patients, $1.46 \pm 0.56 \mathrm{mg} / \mathrm{dL}$ versus $1.09 \pm 0.25 \mathrm{mg} / \mathrm{dL}$ in non-AKI patients, $p<0.0001$. Long-term follow-up for s.Cr values after hospitalization was limited by our hospital being a large referral center. Many patients received their nonsurgical care at local care facilities geographically removed from Mayo Clinic. For those who continued regular nonsurgical followup with us, a persistent separation of s.Cr values between the AKI and non-AKI groups was observed (Figure 2(a)).

Early postoperative mortality rates were dramatically different in AKI versus non-AKI octogenarians. In-hospital 
TABLE 5: (a) Unadjusted Cox proportional hazard ratio for one-year mortality by AKI stage. (b) Adjusted Cox proportional hazard ratio for one-year mortality by AKI stage.

(a)

\begin{tabular}{lcc}
\hline AKI stage & HR $(95 \% \mathrm{CI})$ & $p$ value \\
\hline No AKI & 1 & Reference \\
Stage 1 & $1.04(0.4-2.4)$ & 0.92 \\
Stage 2 & $3.7(0.8-11.4)$ & 0.08 \\
Stage 3 & $15.9(6.9-36.3)$ & $<0.0001$ \\
\hline
\end{tabular}

Unadjusted.

(b)

\begin{tabular}{lcc}
\hline AKI stage & HR $(95 \% \mathrm{CI})$ & $p$ value \\
\hline No AKI & 1 & Reference \\
Stage 1 & $1.0(0.4-2.4)$ & 0.95 \\
Stage 2 & $3.1(0.7-10.3)$ & 0.13 \\
Stage 3 & $13.6(5.4-33.9)$ & $<0.0001$ \\
\hline
\end{tabular}

Adjusted for age and Charlson comorbidity score.

mortality in AKI patients was $>10$-fold higher than the mortality in non-AKI octogenarians, $12.2 \%$ versus $0.9 \%, p=$ 0.0003. 30-day postsurgery mortality for AKI group was $10.2 \%$ versus $0.9 \%$ in non-AKI groups, $p=0.003$. For the 13 AKI patients who required dialysis, $46 \%$ died within 30 days after surgery, $84.6 \%$ died within 1 year, and $92.3 \%$ died within 5 years (Figure 2(b)). Only two of the dialysis patients were able to come off dialysis at the time of discharge.

Late postoperative mortality rates were different between AKI and non-AKI octogenarians. AKI patients showed a 1year mortality of $23.5 \%$ versus $11.7 \%$ in non-AKI patients, $p=$ 0.02 . Figure 2 (c) shows 1-year survival in non-AKI and AKI (Stages 1-3) octogenarians. Kaplan-Meier analysis of survival in non-AKI and AKI octogenarians with a mean follow-up of $3.94 \pm 4.04$ years demonstrates a higher mortality rate in postoperative AKI octogenarians than those without AKI, $p=0.02$ (Figure $2(\mathrm{~d})$ ).

Unadjusted Cox proportional hazard model showed that AKI Stage 3 was a significant contributor to 1-year patient mortality and AKI Stage 2 was nearly significant (Table 5(a)). Cox proportional hazard model adjusted for age and Charlson comorbidity score showed that AKI Stage 3 independently contributed to the 1-year mortality, HR 13.6, 95\% CI: 5.4-33.9, $p<0.0001$ (Table 5(b)).

\section{Comment}

In this study of octogenarians undergoing heart valve replacement surgery, we show that the incidence of postoperative AKI was nearly 50\%. Moreover, AKI occurrence is associated with a prolonged hospital stay, increased need for higher level of care following discharge, persistently reduced kidney function, and reduced short-term and long-term patient survival.

Demand for heart valvular surgery in octogenarians is expected to increase as the population ages $[3-7,16]$. Studies have shown that valve replacement surgeries have acceptable mortality rates in the very elderly $[6,16]$. At Mayo Clinic Rochester, over $16 \%$ of valve replacement surgeries in 2002 and 2003 were performed for octogenarians. Although less invasive transcatheter aortic valve implantation (TAVI) has recently been introduced into practice, there has been wide center-to-center variation in the rates of postprocedural complications (AKI, stroke, and mortality), 4.7 to over $20 \%$ $[17,18]$, and the procedure is limited to the aortic valve replacement. Conventional valve surgery remains the standard of care.

Older age and preexisting CKD are major risk factors for AKI $[19,20]$. AKI in hospitalized patients tends to progress to end-stage renal failure, especially for the elderly [21], and raises mortality. AKI is known to occur after cardiac surgery and is correlated with mortality [22]. Studies in octogenarians undergoing heart valve replacement have been limited. Available studies examine primarily postoperative mortality; when renal impairments are mentioned, no specific definitions, such as AKIN/RIFLE criteria, were applied [6, 7, 23-25]. The 30-day mortality rates in most studies range from 3.4 to $8.5 \%$ after aortic valve replacement $[6,7,14,16,23-28]$ and 18.2 to $18.5 \%$ after mitral valve replacement [5]. Oneyear mortality ranges from 7.1 to $35 \%$. $[6,7,14,25,28]$. The current study, involving all valve replacement surgeries in 209 octogenarians, shows a 30 -day mortality of $\sim 5 \%$ and 1-year mortality of $17 \%$, roughly in line with previous study results. New to the existing literature is that deaths in this cohort of octogenarians occurred almost exclusively in those with AKI (Table 5(a)). Notably, a small preoperative s.Cr difference of $\sim 0.5 \mathrm{mg} / \mathrm{dL}$ between non-CKD and CKD patients reflected an eGFR difference of $\sim 20 \mathrm{~mL} / \mathrm{min} / \mathrm{BSA}$ (from 52.8 to $34.3 \mathrm{~mL} / \mathrm{min} / \mathrm{BSA}$ ) and nearly a doubling of AKI occurrence. Moreover, longer follow-up allowed for long-term outcome assessment, showing a persistently poor kidney function and higher mortality among AKI octogenarians.

Predictors for AKI by univariate analysis in this cohort of octogenarians were a higher Charlson Comorbidity Index, preexisting $\mathrm{CKD}$, prior myocardial infarction, preoperative anemia, surgical duration, intraoperative fluid administration, low intraoperative hemoglobin, and blood transfusion. Stepwise multivariate analysis adjusting for relevant univariate risk factors showed that $\mathrm{CHF}, \mathrm{CKD}$, total intraoperative fluids, and saline infusion are independent predictors for postoperative AKI in this cohort of octogenarians (Tables $4(\mathrm{a})$ and $4(\mathrm{~b})$ ). Excessive intravenous fluids, especially $0.9 \%$ saline, have been associated with the development of postoperative complications and AKI [29-32]. Volume overload in the setting of AKI has been shown to be associated with poor clinical outcomes including mortality [33].

Cox proportional hazard regression was performed to explore survival differences between AKI and non-AKI octogenarians adjusted for age and comorbidity (Table 5). Mortality rates in AKI octogenarians were $~ 10 \%, 25 \%$, and $50 \%$ in 30 days, 1 year, and 5 years, respectively. Although difficult to make a direct comparison, our results are not inferior to the mortality rates in previous studies inclusive of adult AKI patients of all ages showing in-hospital mortality rates of $20-50 \%[10,34,35]$. Among 13 dialysis octogenarians in the current study, $46 \%$ died at 30 days after operation. Despite a 


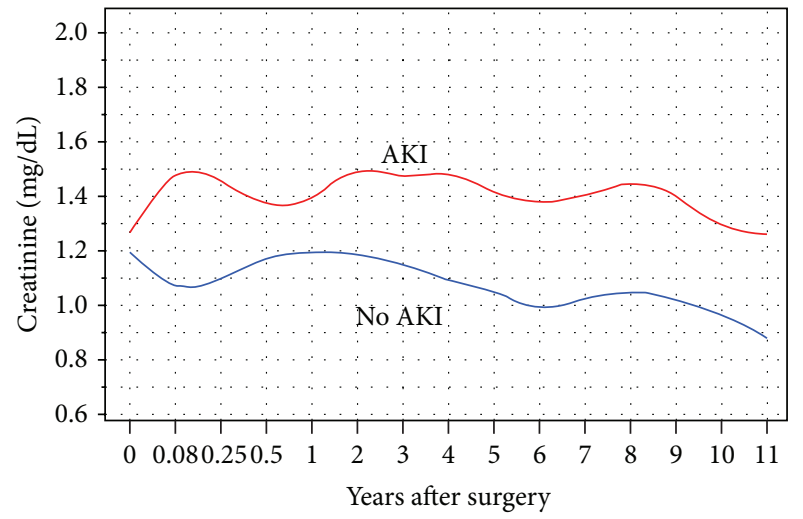

(a)

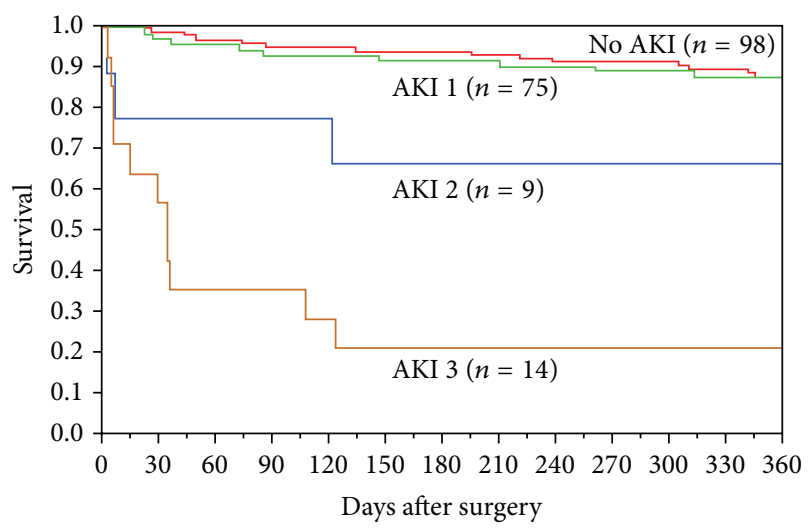

(c)

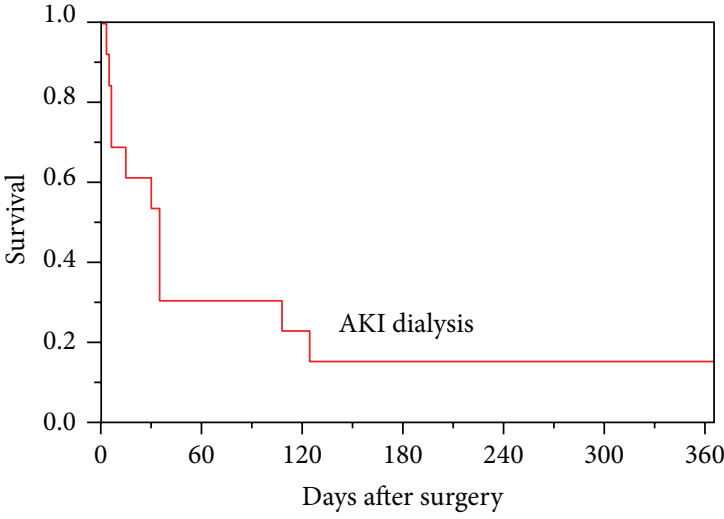

(b)

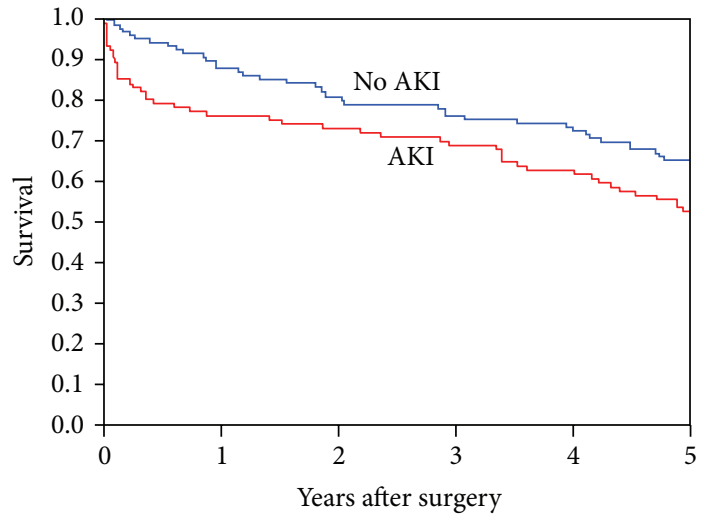

(d)

FIGURE 2: Patient survival. (a) Long-term s.Cr in AKI and non-AKI octogenarians. (b) Kaplan-Meier 1-year survival in octogenarians with AKI requiring dialysis $(n=13)$. (c) Kaplan-Meier 1-year survival by AKI Stages versus non-AKI, unadjusted log-rank $p<0.0001$. (d) KaplanMeier survival curves, AKI versus non-AKI patients, $\log$-rank $p=0.016$. The adjusted ${ }^{\mathrm{a}}$ HR for AKI 2.02; 95\% CI 1.01-4.04, $p=0.04$.

high mortality rate, it is not inferior to the results generated in nonelderly adult AKI-dialysis patients of 55\% [36].

In the current study, concomitant $\mathrm{CABG}$ or number of coronary vessels bypassed did not increase the rate of AKI (Table 2) or mortality, consistent with previous studies showing no mortality impact in concomitant CABG to valve surgery $[5,7]$. There were, however, also studies showing an increase or decrease in mortality in valve surgery with CABG $[14,23,24]$. Given the limited patient number, our results should be viewed with caution. Further study with larger patient number is necessary.

Florath et al. [14] reported mortality rates of $8.4 \%$ at 30 days, $15.2 \%$ at 6 months, and $26 \%$ at 1 year in octogenarians undergoing AVR. An age $>84$ years was related to poor survival. We did not find age $>84$ to have been associated with higher AKI or mortality. This discrepancy could have been due to a small difference in eGFR and similar Charlson Index in our cohorts. Chiappini et al. showed [26] that the predictors for mortality in octogenarians following AVR are poor preoperative $\mathrm{EF}$ and heart failure. Our data did not show these being the significant risks for AKI or mortality, although our study patients might be different from theirs. No studies, however, have explored in detail the relationship between post-valve-surgery AKI and patient's outcomes including disposition, long-term kidney function, and patient survival in octogenarians, as shown in the current study.

Approximately two-thirds of AKI progress to a severe stage [37], which is associated with a further increase in mortality. Nephrology involvement in their care can potentially mitigate progression of AKI and its sequelae. Our results support the early involvement of nephrology in the care of octogenarians with AKI, especially for those with reduced preoperative eGFR and those with a small increase in postsurgery s.Cr $(\geq 0.3 \mathrm{mg} / \mathrm{dL})$. In practice, nephrology referral for octogenarian AKI patients has been low at $20 \%$ [38]. That said, confirmatory evidence of the beneficial effects of nephrology involvement should be further studied.

Several limitations in this study should be considered. First, the nature of the observational cohort study limited consistency in the timing of laboratory testing for creatinine and hemoglobin values, which could influence their values. However, surgical patients are unique in that we knew precisely the time of insult (surgery). Furthermore, a single center study prevented large variation in practice and routine blood draws were relatively fixed in time of day. Second, our data were collected in 2002 and 2003, which may not precisely reflect present-day outcomes. However, there has not been a major practice change in valve replacement surgery, and data 
over an uninterrupted period allowed longitudinal follow-up of patients' outcomes. Third, the majority of our patients are Caucasian, with only one black patient and a few patients of other races, which limits the application of our results to other races. Fourth, AKI patients received more intravenous fluids, which could dilute s.Cr and underestimate AKI severity. However, even with this limitation, we still found significant poor clinical outcomes associated with AKI. Fifth, data on the administration of hydroxyethyl starch (HES) and aprotinin, known to be associated with AKI $[39,40]$, were not analyzed because these agents were used occasionally and unlikely to have affected the results. Lastly, the association between AKI and poor clinical outcomes could potentially be affected by the preexisting CKD. The strong effect of CKD may hinder the determination of true impact of AKI on patients' outcomes. Nonetheless, severe AKI stood out as an independent risk factor for mortality in our cohort. We believe that even mild-to-moderate degrees of AKI could potentially exert negative impact on patient survival, although the signal may have been overwhelmed by the extraordinarily strong effect of preexisting CKD. Taken together, CKD is a strong reason for increased mortality after AKI, but AKI independently contributed to the poor patient outcomes.

Overall, our data show that octogenarians are able to tolerate heart valve replacement surgery. However, the AKI occurrence rate is substantial and AKI impacts patients' mortality and morbidity. Better patient selection, preoperative preparation, and avoidance of operative risk factors can minimize the AKI risk and adverse consequences.

\section{Conflict of Interests}

The authors declare that there is no conflict of interests regarding the publication of this paper.

\section{References}

[1] United Nations DoEaSA and Population Division, World Population Ageing 2013, United Nations Publication, 2013.

[2] J. M. Ortman, V. A. Velkoff, and H. Hogan, "An aging nation: the older population in the United States," Tech. Rep. P25-1140, United States Census Bureau, 2014.

[3] V. T. Nkomo, J. M. Gardin, T. N. Skelton, J. S. Gottdiener, C. G. Scott, and M. Enriquez-Sarano, "Burden of valvular heart diseases: a population-based study," The Lancet, vol. 368, no. 9540, pp. 1005-1011, 2006.

[4] G. Asimakopoulos, M.-B. Edwards, and K. M. Taylor, "Aortic valve replacement in patients 80 years of age and older: Survival and cause of death based on 1100 cases-collective results from the UK heart valve registry," Circulation, vol. 96, no. 10, pp. 3403-3408, 1997.

[5] J. Nloga, R. Hénaine, M. Vergnat et al., "Mitral valve surgery in octogenarians: should we fight for repair? A survival and quality-of-life assessment," European Journal of Cardio-thoracic Surgery, vol. 39, no. 6, pp. 875-880, 2011.

[6] D. Calvo, I. Lozano, J. C. Llosa et al., "Aortic valve replacement in octogenarians with severe aortic stenosis. Experience in a series of consecutive patients at a single center," Revista Espanola de Cardiologia, vol. 60, no. 7, pp. 720-726, 2007.
[7] J. A. Ditchfield, E. Granger, P. Spratt et al., "Aortic valve replacement in octogenarians," Heart Lung and Circulation, vol. 23, no. 9, pp. 841-846, 2014.

[8] R. Prêtre and M. I. Turina, "Cardiac valve surgery in the octogenarian," Heart, vol. 83, no. 1, pp. 116-121, 2000.

[9] T. Z. Ali, I. Khan, W. Simpson et al., "Incidence and outcomes in acute kidney injury: a comprehensive population-based study," Journal of the American Society of Nephrology, vol. 18, no. 4, pp. 1292-1298, 2007.

[10] S. Uchino, R. Bellomo, D. Goldsmith, S. Bates, and C. Ronco, "An assessment of the RIFLE criteria for acute renal failure in hospitalized patients," Critical Care Medicine, vol. 34, no. 7, pp. 1913-1917, 2006.

[11] M. E. Charlson, P. Pompei, K. A. Ales, and C. R. MacKenzie, "A new method of classifying prognostic comorbidity in longitudinal studies: development and validation," Journal of Chronic Diseases, vol. 40, no. 5, pp. 373-383, 1987.

[12] H. S. Kilbride, P. E. Stevens, G. Eaglestone et al., "Accuracy of the MDRD (Modification of Diet in Renal Disease) study and CKD-EPI (CKD Epidemiology Collaboration) equations for estimation of GFR in the elderly," American Journal of Kidney Diseases, vol. 61, no. 1, pp. 57-66, 2013.

[13] R. L. Mehta, J. A. Kellum, S. V. Shah et al., "Acute Kidney Injury Network: report of an initiative to improve outcomes in acute kidney injury," Critical Care, vol. 11, no. 2, article R31, 2007.

[14] I. Florath, A. Albert, A. Boening, I. C. Ennker, and J. Ennker, "Aortic valve replacement in octogenarians: identification of high-risk patients," European Journal of Cardio-Thoracic Surgery, vol. 37, no. 6, pp. 1304-1310, 2010.

[15] L. Henry, L. Halpin, S. Hunt, S. D. Holmes, and N. Ad, "Patient disposition and long-term outcomes after valve surgery in octogenarians," Annals of Thoracic Surgery, vol. 94, no. 3, pp. 744-750, 2012.

[16] T. Langanay, E. Flécher, O. Fouquet et al., "Aortic valve replacement in the elderly: the real life," Annals of Thoracic Surgery, vol. 93, no. 1, pp. 70-78, 2012.

[17] M. Rahnavardi, J. Santibanez, K. Sian, and T. D. Yan, "A systematic review of transapical aortic valve implantation," Annals of Cardiothoracic Surgery, vol. 1, no. 2, pp. 116-128, 2012.

[18] D. Dvir, J. G. Webb, S. Bleiziffer et al., "Transcatheter aortic valve implantation in failed bioprosthetic surgical valves," The Journal of the American Medical Association, vol. 312, no. 2, pp. 162-170, 2014.

[19] K. Nash, A. Hafeez, and S. Hou, "Hospital-acquired renal insufficiency," American Journal of Kidney Diseases, vol. 39, no. 5, pp. 930-936, 2002.

[20] C.-Y. Hsu, C. E. McCulloch, D. Fan, J. D. Ordoñez, G. M. Chertow, and A. S. Go, "Community-based incidence of acute renal failure," Kidney International, vol. 72, no. 2, pp. 208-212, 2007.

[21] A. Ishani, J. L. Xue, J. Himmelfarb et al., "Acute kidney injury increases risk of ESRD among elderly," Journal of the American Society of Nephrology, vol. 20, no. 1, pp. 223-228, 2009.

[22] A. Ishani, D. Nelson, B. Clothier et al., "The magnitude of acute serum creatinine increase after cardiac surgery and the risk of chronic kidney disease, progression of kidney disease, and death," Archives of Internal Medicine, vol. 171, no. 3, pp. 226-233, 2011.

[23] K. P. Alexander, K. J. Anstrom, L. H. Muhlbaier et al., "Outcomes of cardiac surgery in patients age $\geq 80$ years: results from the national cardiovascular network," Journal of the American College of Cardiology, vol. 35, no. 3, pp. 731-738, 2000. 
[24] S. J. Melby, A. Zierer, S. P. Kaiser et al., "Aortic valve replacement in octogenarians: risk factors for early and late mortality," The Annals of Thoracic Surgery, vol. 83, no. 5, pp. 1651-1657, 2007.

[25] V. H. Thourani, R. Myung, P. Kilgo et al., "Long-term outcomes after isolated aortic valve replacement in octogenarians: a modern perspective," Annals of Thoracic Surgery, vol. 86, no. 5, pp. 1458-1465, 2008.

[26] B. Chiappini, N. Camurri, A. Loforte, L. Di Marco, R. Di Bartolomeo, and G. Marinelli, "Outcome after aortic valve replacement in octogenarians," Annals of Thoracic Surgery, vol. 78, no. 1, pp. 85-89, 2004.

[27] A. Mortasawi, S. Gehle, M. Yaghmaie et al., "Short and long term results of aortic valve replacement in patients 80 years of age and older," Herz, vol. 26, no. 2, pp. 140-148, 2001.

[28] G. Rizzoli, J. Bejko, T. Bottio, V. Tarzia, and G. Gerosa, "Valve surgery in octogenarians: does it prolong life?" European Journal of Cardio-Thoracic Surgery, vol. 37, no. 5, pp. 1047-1055, 2010.

[29] N. M. Yunos, R. Bellomo, F. C. Hegarty, D. Story, L. Ho, and M. Bailey, "Association between a chloride-liberal vs chloriderestrictive intravenous fluid administration strategy and kidney injury in critically ill adults," Journal of the American Medical Association, vol. 308, no. 15, pp. 1566-1572, 2012.

[30] J. A. Kellum, "Fluid resuscitation and hyperchloremic acidosis in experimental sepsis: improved short-term survival and acidbase balance with Hextend compared with saline," Critical Care Medicine, vol. 30, no. 2, pp. 300-305, 2002.

[31] B. A. Cotton, J. S. Guy, J. A. Morris Jr., and N. N. Abumrad, “The cellular, metabolic, and systemic consequences of aggressive fluid resuscitation strategies," Shock, vol. 26, no. 2, pp. 115-121, 2006.

[32] B. Brandstrup, H. Tønnesen, R. Beier-Holgersen et al., "Effects of intravenous fluid restriction on postoperative complications: comparison of two perioperative fluid regimens: a randomized assessor-blinded multicenter trial," Annals of Surgery, vol. 238, no. 5, pp. 641-648, 2003.

[33] J. Bouchard, S. B. Soroko, G. M. Chertow et al., "Fluid accumulation, survival and recovery of kidney function in critically ill patients with acute kidney injury," Kidney International, vol. 76, no. 4, pp. 422-427, 2009.

[34] Y. Fang, X. Ding, Y. Zhong et al., "Acute kidney injury in a chinese hospitalized population," Blood Purification, vol. 30, no. 2, pp. 120-126, 2010.

[35] J.-P. Lafrance and D. R. Miller, "Acute kidney injury associates with increased long-term mortality," Journal of the American Society of Nephrology, vol. 21, no. 2, pp. 345-352, 2010.

[36] C. V. Thakar, S. Worley, S. Arrigain, J.-P. Yared, and E. P. Paganini, "Improved survival in acute kidney injury after cardiac surgery," American Journal of Kidney Diseases, vol. 50, no. 5, pp. 703-711, 2007.

[37] E. A. J. Hoste, G. Clermont, A. Kersten et al., "RIFLE criteria for acute kidney injury are associated with hospital mortality in critically ill patients: a cohort analysis," Critical Care, vol. 10, no. 3, article R73, 2006.

[38] T. Ali, A. Tachibana, I. Khan et al., "The changing pattern of referral in acute kidney injury," QJM, vol. 104, no. 6, pp. 497503, 2011.

[39] J. A. Myburgh, S. Finfer, R. Bellomo et al., "Hydroxyethyl starch or saline for fluid resuscitation in intensive car," The New England Journal of Medicine, vol. 367, no. 20, pp. 1901-1911, 2012.
[40] D. T. Mangano, I. C. Tudor, and C. Dietzel, “The risk associated with aprotinin in cardiac surgery," The New England Journal of Medicine, vol. 354, no. 4, pp. 353-365, 2006. 


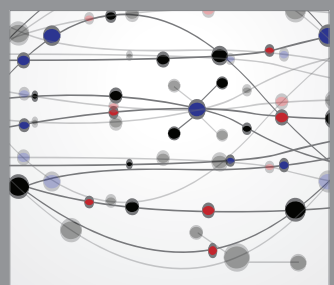

The Scientific World Journal
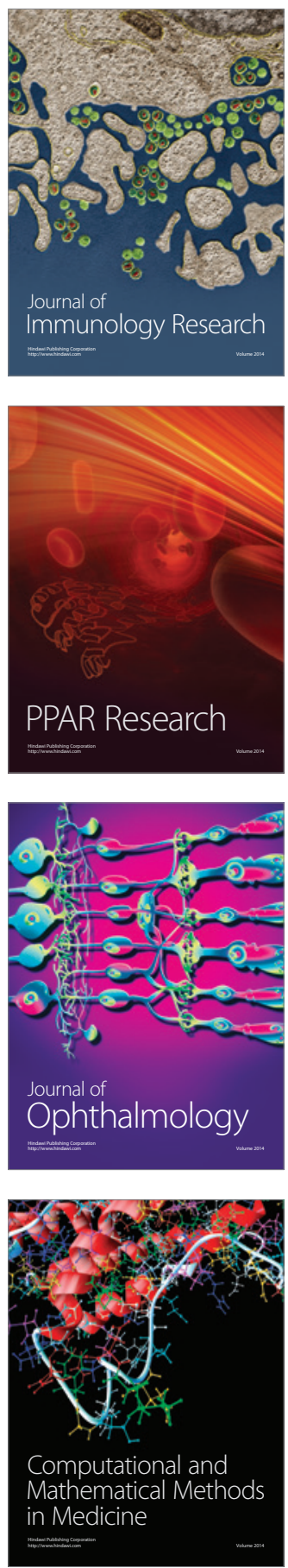

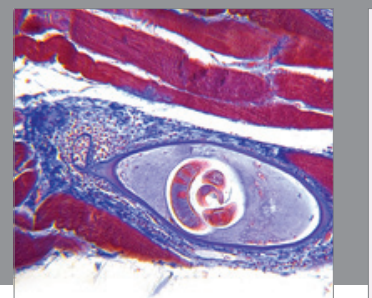

Gastroenterology

Research and Practice
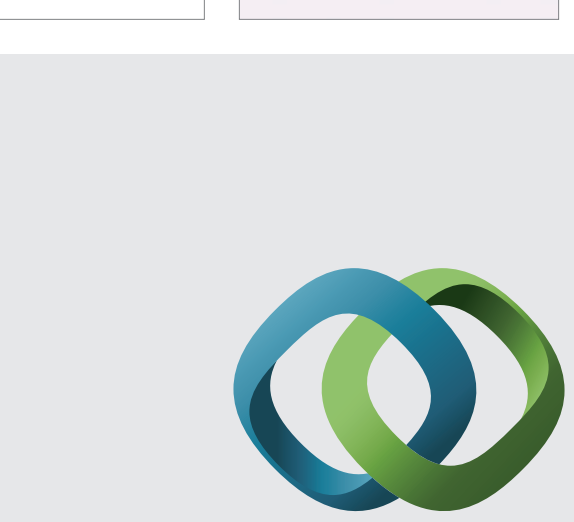

\section{Hindawi}

Submit your manuscripts at

http://www.hindawi.com
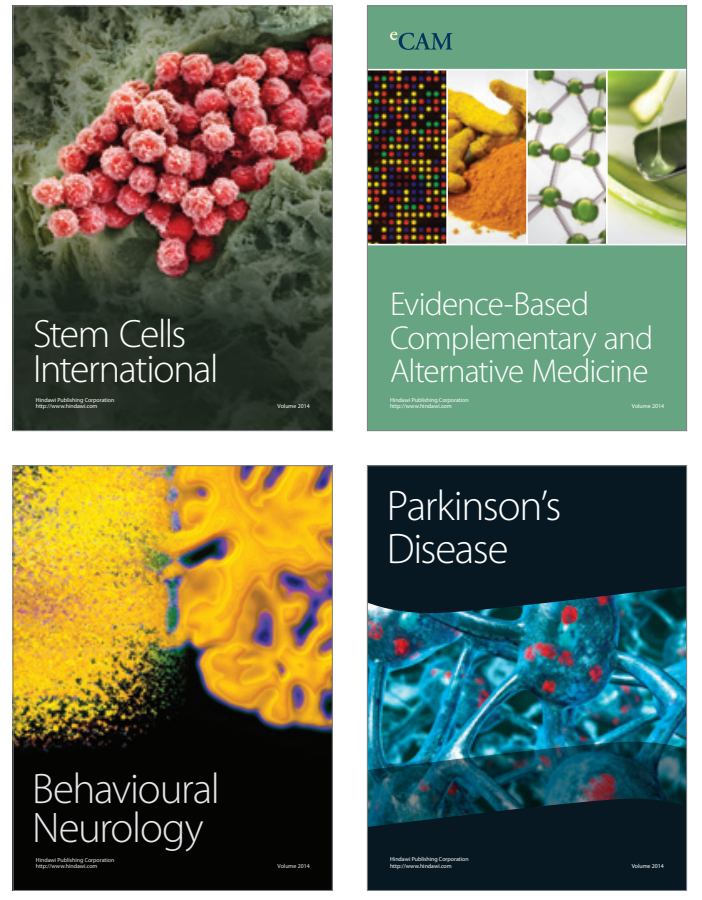
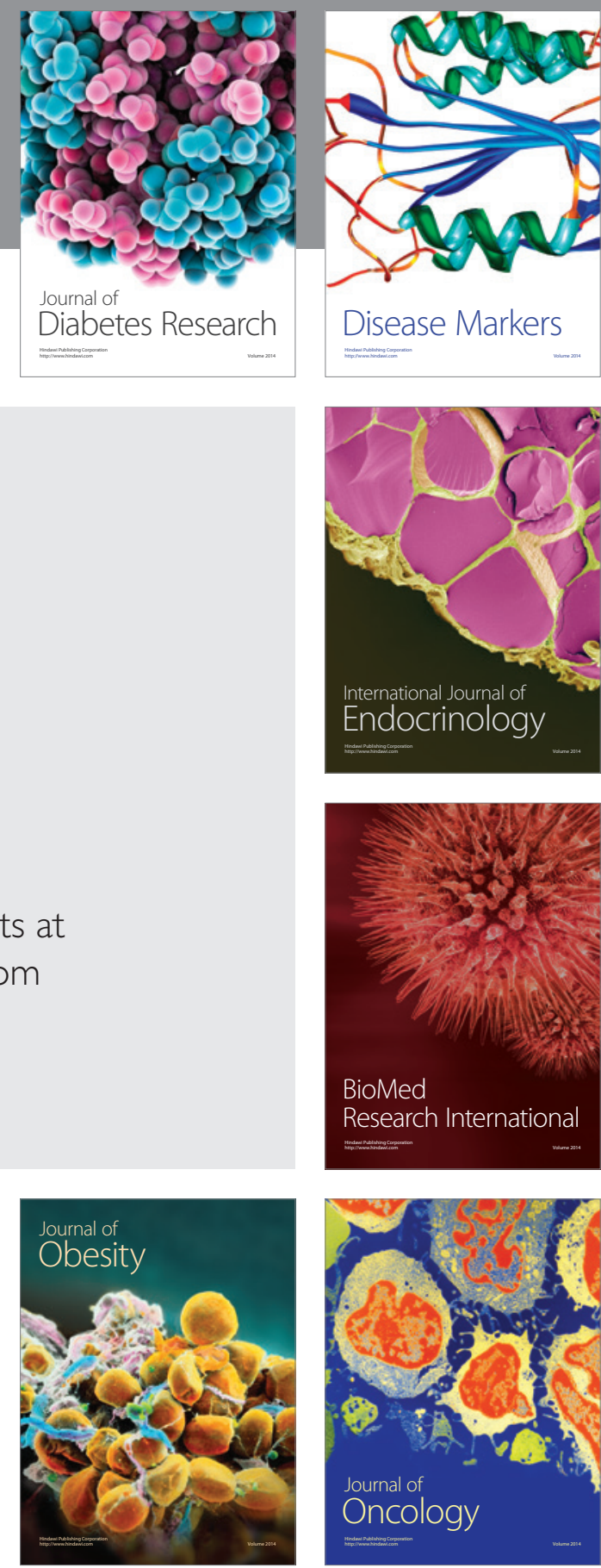

Disease Markers
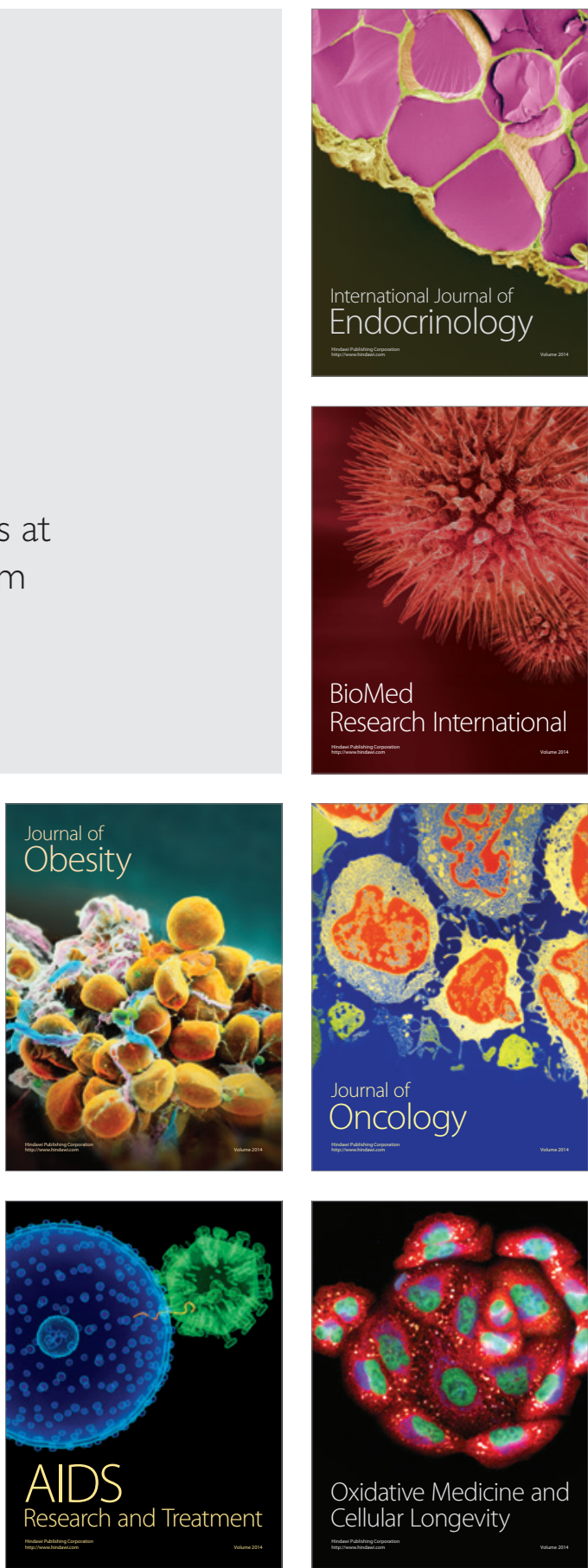